\title{
Zusammenfassung Teil I
}

Der erste Teil dieses Buches hat einen umfassenden Überblick über die Technologien der Mixed Reality vermittelt. Sowohl AR als auch VR lassen sich bei einer Vielzahl von Anwendungsfällen entlang der Wertschöpfungskette produzierender Unternehmen nutzen. Ziel war es, Unternehmen einen Orientierungsrahmen für das systematische Identifizieren von Ansatzpunkten für eigene Geschäftsmodell-Innovation mit XR zu bieten. Beispiele aus Literatur und Praxis haben die Geschäftsmodellmuster konkretisiert.

In der Rückschau wird bei Geschäftsmodellmustern wie „Guaranteed Availability“, „Direct Selling“ oder „Digitalization“ deutlich, dass XR zum einen einzelne Elemente eines Geschäftsmodells verändern kann, indem beispielsweise Prozesse optimiert werden. Zum anderen lassen sich Kostensenkungspotenziale durch eine Reduktion von Prototyp-, Reise-, Marketing- oder Beschaffungskosten erschließen. Weitere Geschäftsmodellmuster wie etwa „Cash Maschine“ oder „From-Push-to-Pull“ können nicht nur Kosten bei der Leistungserstellung senken, sondern ebenso neue Ertragsquellen erschließen.

Der nachfolgende Teil II stellt eine Vorgehensweise vor, wie produzierende Unternehmen konkrete Geschäftsmodelle auf Basis von Technologien der Mixed Reality umsetzen können. 